Original Article

\title{
Comparison of the Incidence of Complications and Secondary Surgical Interventions Necessary in Patients with Chronic Lower Limb Ischemia Treated by Both Open and Endovascular Surgeries
}

\author{
Dariusz Janczak, MD, PhD, ${ }^{1,2}$ Maciej Malinowski, MD, ${ }^{1,2}$ Wojciech Bąkowski, ${ }^{2}$ \\ Katarzyna Krakowska, ${ }^{2}$ Karol Marschollek, ${ }^{2}$ Paweł Marschollek, ${ }^{2}$ \\ and Mariusz Chabowski, MD, $\mathrm{PhD}^{1,2}$
}

\begin{abstract}
Background: Peripheral arterial disease (PAD) affects 3\%-10\% of the population before the age of 70 years and $15 \%-20 \%$ after that age.

The aim of the study was to compare the incidence of complications and secondary interventions in patients who underwent each type of treatment.

Methods: We analyzed 734 medical records of the Department of Surgery at the 4th Military Teaching Hospital in Wroclaw, In total, 394 were operated on with open surgery; an endarterectomy $(59.39 \%)$, a vascular prosthesis implantation $(31.01 \%)$, or both of these techniques $(6.6 \%)$, and 340 patients had angioplasty with $(50.59 \%)$ or without stenting $(49.41 \%)$.

Results: There were no statistically significant differences in the incidence of corresponding complications. The exception was the infection of the wound; significantly fewer were reported in the case of endovascular procedures $(p=0.0087)$. There were 12 occasions $(3.53 \%)$ during endovascular surgeries when intraoperative conversion or re-operation using the open method occurred. In the case of open surgery, the mean hospital stay was 7.77 days (median: 8, mode: 8), while for endovascular management it was equal to 4.68 days (median: 4, mode: 3), $\mathrm{p}<\mathbf{0 . 0 0 0 1}$.

Conclusion: The endovascular method results in a similar re-operation rate and number of complications as open surgery.
\end{abstract}

Keywords: peripheral arterial occlusive disease, chronic lower limb ischemia, open surgery, endovascular surgery, complications

\footnotetext{
${ }^{1}$ Division of Surgical Specialties, Department of Clinical Nursing, Faculty of Health Science, Wroclaw Medical University, Wroclaw, Poland

${ }^{2}$ Department of Surgery, 4th Military Teaching Hospital, Wroclaw, Poland

Received: November 28, 2016; Accepted: January 21, 2017 Corresponding author: Mariusz Chabowski, MD, PhD. Department of Surgery, 4th Military Teaching Hospital, 5 Weigla Street, 50-981 Wroclaw, Poland.

Email: mariusz.chabowski@gmail.com

(C)2017 The Editorial Committee of Annals of Thoracic and Cardiovascular Surgery. All rights reserved.
}

\section{Introduction}

More than $98 \%$ of lower extremity arteries stenoses occur due to an atherosclerosis, leading to progressive ischemia of the legs which consequently may cause necrosis and limb loss. ${ }^{1)}$ The most common symptoms encountered by the patient are easy leg fatigue, paresthesia, intermittent claudication, and rest pain. However, the patient may not report any of these symptoms. Signs such as blue or pale skin on the limb or lack of pulse 
below the obstruction may also be observed. Epidemiological studies show that $3 \%-10 \%$ of the population suffers from chronic ischemia of the lower limbs (peripheral arterial disease [PAD]), and this percentage increases with age: over the age of 70 years, the disease is already present in $15 \%-20 \%$ of the population. The authors of TransAtlantic InterSociety II Consensus (TASC II) also indicate that in patients with critical limb ischemia (CLI), $20 \%$ of them die within the first year after diagnosis. ${ }^{2)}$ A high mortality and a worse prognosis are associated with the frequent co-occurrence of hypertension, ischemic heart disease, chronic kidney disease, and diabetes mellitus type II. ${ }^{3)}$ In the case of less severe lowerextremity atherosclerosis, a conservative treatment is the first choice. An important part of it is a reduction of the parameters considered as risk factors for PAD: high blood pressure, hypercholesterolemia, or hyperhomocysteinemia. Attention is also drawn to elevated levels of inflammatory markers, especially $\alpha$-defensins and C-reactive protein in patients at increased risk. ${ }^{4)}$ It is believed that chronic cigarette smoking is a greater risk factor for PAD than for coronary heart disease. ${ }^{5)}$ In patients with more severe lesions and shorter claudication distance, surgical intervention is necessary. A choice of the best method of treatment is becoming more difficult and requires specific diagnostics. It is essential to determine the location and extent of the changes, as well as to analyze the patient's general state together with any chronic diseases and presented symptoms. At present, two methods are most commonly used in the treatment of patients with PAD.

Open surgery is a longer practiced and thus better known procedure, which consists of performing an endarterectomy, placing prosthesis or vascular patches in place of damaged vessel, ensuring its continuity and patency. This method is deemed as being highly invasive. A newer method is percutaneous transluminal angioplasty (PTA), first performed in 1964 at Oregon Health Science University by Charles T. Dotter and his intern Melvin P. Judkins. It involves the percutaneous introduction of a catheter balloon into an appropriate artery, which, in the narrowed part of the artery, expands by filling with a contrast agent. The process is observed by intra-operative X-ray. PTA is much less invasive, leading to an essentially shorter hospital stay. According to Polish recommendations, published in 2009 and established in accordance with the guidelines of TASC II, only changes assigned to category D are clearly not to be managed using endovascular procedures. ${ }^{6)}$ Depending on the location they are classified as

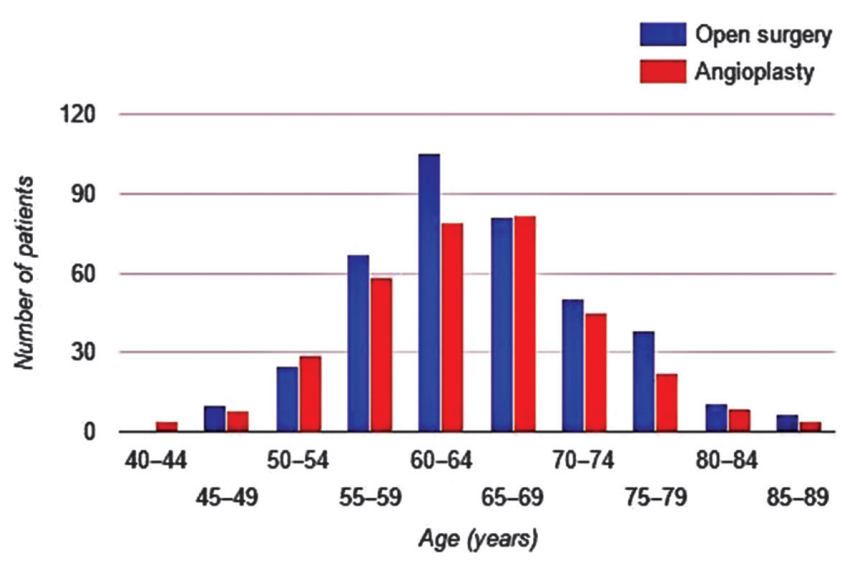

Fig. 1 Patients' distribution by age.

following: an aorto-iliac section: an infrarenal aorto-iliac occlusion, diffuse changes involving both the aorta and iliac arteries, multiple unilateral stenoses, bilateral occlusion of the iliac arteries, an accompanying infrarenal abdominal aortic aneurysm; a femoro-popliteal segment: chronic total occlusion of the common femoral, superficial femoral or popliteal artery; and tibial arteries: a lack of patent collateral circulation. Other conditions may be treated either using the open or endovascular method.

The aim of the study was to compare the incidence of complications and the necessity for re-operation within 6 months of the initial surgery. The open and endovascular methods were compared.

\section{Materials and Methods}

We retrospectively analyzed 734 medical records from the Department of Surgery at the 4th Military Teaching Hospital in Wroclaw. The patients were treated for chronic lower limb ischemia in the course of atherosclerosis obliterans from October 2010 to December 2014. The patients' distribution by age is shown in Fig. 1 . Hospitalized patients were subjected to a diagnostic process before surgery in order to determine the severity of lesions. Patients were qualified for angiosurgical treatment on the base of imaging methods (carotid duplex ultrasound [CDUS], computed tomography angiography [CTA], and digital subtraction angiography [DSA]) and clinical symptoms assessed with the Rutherford classification. Endovascular management was preferred as a first-line therapy. Patients, in whom the PTA was unsuccessful or preliminary medical imaging had predicted a failure of angioplasty, were treated with open surgeries. The distributions of lesions' location and TASC classification are presented in Tables $\mathbf{1}$ and $\mathbf{2}$. For surgical 
Table 1 The distribution of lesions' location in both the analyzed groups

\begin{tabular}{lcc}
\hline \multirow{2}{*}{ Location } & \multicolumn{2}{c}{ Percentage } \\
\cline { 2 - 3 } & $\begin{array}{c}\text { Open surgeries } \\
(\%)\end{array}$ & $\begin{array}{c}\text { Endovascular } \\
\text { surgeries }(\%)\end{array}$ \\
\hline Aorto-iliac & 11.29 & 16.67 \\
Femoro-popliteal & 47.58 & 55.83 \\
Multilevel & 41.13 & 27.50 \\
\hline
\end{tabular}

Table 2 TASC qualification of vascular lesions in the groups managed with open and endovascular surgeries

\begin{tabular}{lcc}
\hline & \multicolumn{2}{c}{ Percentage } \\
\cline { 2 - 3 } Type & $\begin{array}{c}\text { Open surgeries } \\
(\%)\end{array}$ & $\begin{array}{c}\text { Endovascular surgeries } \\
(\%)\end{array}$ \\
\hline $\mathrm{A}$ & 1.61 & 24.17 \\
$\mathrm{~B}$ & 21.77 & 36.67 \\
$\mathrm{C}$ & 25.81 & 25.83 \\
$\mathrm{D}$ & 50.81 & 13.33 \\
\hline
\end{tabular}

TASC: TransAtlantic InterSociety Consensus

treatment, patients with CLI were qualified: rest pain (Rutherford classification IV) was present in $24.81 \%$ for open and $18.53 \%$ for endovascular surgeries; tissue loss (Rutherford classification V-VI) was claimed in $32.97 \%$ and $14.68 \%$, respectively, for open and endovascular treatment. There were also managed patients with severe intermittent claudication (Rutherford classification III), if the symptoms significantly impair their normal activity. In open surgeries, it was $42.22 \%$ of patients, and in endovascular, it was $66.79 \%$. Out of all, 394 were treated by open surgery and 340 by PTA. In patients operated on conventionally, an endarterectomy (59.39\%), vascular prosthesis implantation $(34.01 \%)$, or both techniques $(6.6 \%)$ were performed. In patients undergoing endovascular surgery, a balloon angioplasty with $(50.59 \%)$ or without stenting $(49.41 \%)$ was carried out.

In the group of patients who underwent open surgery, the number of women amounted to $132(33.5 \%)$ and the number of men totaled $262(66.5 \%)$. In the group treated by angioplasty, the number of women was 111 (32.65\%) and the number of men stood at $229(67.35 \%)$. Using the chi-squared test, the uniformity of the compared groups in terms of sex was determined $(p=0.868)$. The mean age of the patients treated by open surgery was equal to 65 years, and endovascularly, it was equal to 64 years. The Student's t-test for independent groups showed the uniformity of age structure in the groups under analysis $(p=0.267)$.
The incidence of comorbidities in both groups was also taken into consideration. Table 3 contains this statement. It should be mentioned, however, that in the case of nicotinism the percentage of patients in both groups may have been underestimated due to insufficient medical data concerning stimulants. The incidence of complications was statistically evaluated using Fisher's exact test, while the average length of hospitalization was compared using the t-test for independent groups. The established level of significance was $\alpha=0.05$.

All the patients provided written informed consent to have their data used in this study. This study was approved by the Medical University Ethics Committee.

\section{Results}

The registered complications and the number of surgical interventions performed within 6 months of the initial surgery in patients treated by open and endovascular methods are shown in Table 4. A comparative analysis of both groups showed no statistically significant differences in the incidence of corresponding complications. Only in the case of wound infection, it was revealed that there was a significantly lower number of them in the group of endovascular procedures $(\mathrm{p}=0.0087)$. During 12 angioplasty procedures $(3.53 \%)$, intraoperative conversion or re-operation using the open method occurred.

We also compared the average length of hospitalization of patients in both groups. In the group of open surgery, it amounted to 7.77 days (median: 8, mode: 8), while for endovascular treatment, it equaled 4.68 days (median: 4, mode: 3). The statistical significance was determined as $\mathrm{p}<0.0001$. Patients who did not have postoperative complications were discharged from the hospital with improved flow and blood supply to the lower limbs. The increasing popularity of endovascular procedures is also visible in the Department of Surgery at the 4th Military Teaching Hospital in Wrocław. While in 2012, approximately 100 operations were performed using this method (34\% of all treatment), in 2014 there were about 180 of them, accounting for almost $58 \%$ of all surgical interventions in patients with chronic ischemia of the lower limbs (Fig. 2).

\section{Discussion}

The increasing popularity of endovascular surgery is the reason why the debate on the appropriate choice of a method for surgical treatment of CLI is controversial. 
Table 3 The most common comorbidities recorded in patients treated by open and endovascular surgeries

\begin{tabular}{lcc}
\hline Concomitant disease & $\begin{array}{c}\text { The number of patients in the group } \\
\text { treated by open surgery }\end{array}$ & $\begin{array}{c}\text { The number of patients in the group treated } \\
\text { by endovascular surgery }\end{array}$ \\
\hline Ischemic heart disease & $109(27.7 \%)$ & $77(22.6 \%)$ \\
Noninsulin-dependent diabetes mellitus & $100(25.4 \%)$ & $84(24.7 \%)$ \\
History of myocardial infarction & $64(16.2 \%)$ & $58(17 \%)$ \\
History of stroke & $25(6.3 \%)$ & $29(8.5 \%)$ \\
Heart failure & $22(5.6 \%)$ & $13(3.8 \%)$ \\
COPD & $19(4.8 \%)$ & $20(5.9 \%)$ \\
Permanent atrial fibrillation & $15(3.8 \%)$ & $12(3.5 \%)$ \\
Chronic kidney disease & $13(3.3 \%)$ & $24(7 \%)$ \\
Alcohol use disorder & $13(3.3 \%)$ & $9(2.6 \%)$ \\
Smoking (ever) & $197(50 \%)$ & $223(65.6 \%)$ \\
\hline
\end{tabular}

COPD: chronic obstructive pulmonary disease

Table 4 The number of complications in patients treated using open surgery $(n=394)$ and angioplasty $(n=340)$

\begin{tabular}{|c|c|c|c|}
\hline Complication & Open surgery & Endovascular angioplasty & Statistical significance $(\mathrm{p})$ \\
\hline $\begin{array}{l}\text { Re-ischemia of the operated limb requiring } \\
\text { surgical intervention }\end{array}$ & $26(6.60 \%)$ & $16(4.71 \%)$ & 0.339222 \\
\hline Infection of the surgical wound & $16(4.06 \%)$ & $3(0.88 \%)$ & $0.008681^{*}$ \\
\hline Amputation & $9(2.28 \%)$ & $2(0.59 \%)$ & 0.071302 \\
\hline $\begin{array}{l}\text { Re-ischemia of the opposite to operated limb } \\
\text { requiring surgical intervention }\end{array}$ & $6(1.52 \%)$ & $3(0.88 \%)$ & 0.515825 \\
\hline Bleeding from the wound, hematoma & $4(1.02 \%)$ & $6(1.76 \%)$ & 0.52633 \\
\hline Death & $4(1.02 \%)$ & $0(0 \%)$ & 0.128041 \\
\hline Limb edema & $3(0.76 \%)$ & $1(0.29 \%)$ & 0.628045 \\
\hline Peroneal nerve palsy & $2(0.51 \%)$ & $0(0 \%)$ & 0.502028 \\
\hline Retroperitoneal hematoma & $2(0.51 \%)$ & $1(0.29 \%)$ & 1 \\
\hline Hemorrhage to the gastrointestinal tract & $2(0.51 \%)$ & $0(0 \%)$ & 0.502028 \\
\hline Lymphorrhea & $2(0.51 \%)$ & $0(0 \%)$ & 0.502028 \\
\hline False aneurysm & $2(0.51 \%)$ & $2(0.59 \%)$ & 1 \\
\hline Dyspnea & $2(0.51 \%)$ & $0(0 \%)$ & 0.502028 \\
\hline Respiratory failure & $1(0.25 \%)$ & $0(0 \%)$ & 1 \\
\hline Circulatory failure & $1(0.25 \%)$ & $0(0 \%)$ & 1 \\
\hline Psychotic symptoms & $1(0.25 \%)$ & $0(0 \%)$ & 1 \\
\hline Wound dehiscence & $1(0.25 \%)$ & $0(0 \%)$ & 1 \\
\hline Deep vein thrombosis & $0(0 \%)$ & $1(0.29 \%)$ & 0.463215 \\
\hline
\end{tabular}

*The differences in complications incidence between both groups are significant with $\mathrm{p}<0.05^{\prime \prime}$

Published in 2007, TASC II includes recommendations for invasive treatment of PAD and makes the choice between open and endovascular surgeries dependent on the location and the extent of vascular lesions. In many cases, the decision is also based on other factors, such as age and the patient's general condition. According to the guidelines, type A changes are indicative of endovascular treatment, while in changes categorized as types B and $\mathrm{C}$ such treatment can be applied. However, endovascular treatment is not considered suitable for category $\mathrm{D}$ changes. ${ }^{2)}$

The aim of our study was to compare the incidence of complications and of the necessity to re-operate on patients operated on by the two methods: open surgery and angioplasty. The available literature does not provide clear answers to the problems addressed in our research. In the multicenter, randomized trial bypass versus angioplasty in severe ischaemia of the leg (BASIL), patients diagnosed with CLI were divided by the implemented method of surgical treatment. ${ }^{7,8)}$ The results showed no statistically significant differences between the endovascular and open methods, both in the number of amputations performed within 6 months after surgery and in the patients' declared quality of life. At the same time, it indicated an increased incidence of early complications (within 30 days) and higher costs associated with open surgery. However, long-term observations show greater survival rates among patients undergoing classic surgery. 


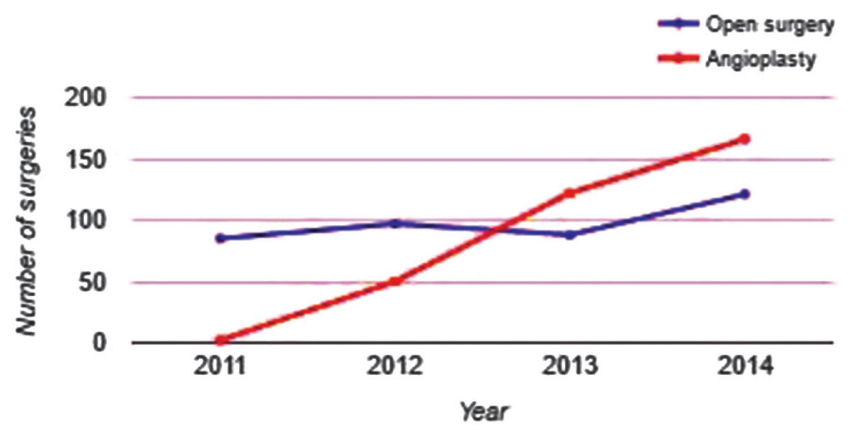

Fig. 2 Changes in the numbers of operations performed using the open and endovascular methods at the 4th Military Teaching Hospital in Wroclaw for the years from 2011 to 2014.

Dosologlu et al. reported comparable results of the hemodynamic effects and the number of amputations required in the long term. The authors also highlight the fact that the endovascular method was preferred in patients in a more severe clinical condition, whereas patients with more extensive changes were recommended for open treatment.9)

Cull et al. and Suding et al. note that, in the case of endovascular procedures, the number of performed amputations did not significantly change. ${ }^{10,11)}$ However, Cull et al. argue that the amount of necessary reinterventions is higher compared to open surgery. ${ }^{10)}$

A similar percentage of postoperative complications reported in this analysis in our opinion justifies focusing on other advantages and disadvantages of both techniques when selecting the most appropriate method for a particular patient. The proper qualification of patients for treatment has a crucial impact on achieving good results, a fact which is also emphasized by other authors. ${ }^{5,12,13)}$

Bodzon et al. reported that patients with advanced limb ischemia, not qualified for open surgery, can be treated using angioplasty with satisfactory results as long as the nature of the atherosclerotic lesions permits such management. ${ }^{14)}$

In the case of relatively young patients being qualified for angioplasty, we should consider the fact that there is a smaller amount of data available on the long-term complications. The limited usefulness of this method in this particular group of patients is also highlighted by Morbi et al. ${ }^{15}$ )

The differences in the incidence of complications in the two groups are not statistically significant. Only the infection of the wound occurred significantly more often in patients undergoing open surgery, which can be associated with the greater invasiveness of the technique.
The average age of patients in both groups was similar. It can be noted that the age distribution of patients treated with angioplasty has shifted to the right, towards higher values, compared to the corresponding distribution in the group treated by open surgery. Also, the presence of comorbidities associated with patients in both groups stands at a similar level. Among those that could have a considerable impact on the course of treatment, patients undergoing endovascular surgery were diagnosed with chronic kidney disease significantly more frequently.

Hospitalization time was remarkably shorter (60.8\%) during endovascular procedures. Other authors represent similar results. ${ }^{8,16-18)}$ The longer hospitalization associated with open surgery is related to higher costs and reduced comfort of the patients and their families.

\section{Conclusion}

We conclude that the endovascular method leads to a similar re-operation rate and number of complications as open surgery. Angioplasty is becoming increasingly more popular and more frequently used. The severe condition of a patient, their age, plus any concomitant diseases are all indications that point to considering an endovascular procedure so that the treatment is less invasive and the results of the treatment are maintained at an appropriate level.

\section{Authors' Contributions}

Dariusz Janczak: research concept and design

Wojciech Bąkowski, Katarzyna Krakowska, Karol Marschollek, and Paweł Marschollek: collection and/or assembly of data

Wojciech Bąkowski and Karol Marschollek: data analysis and interpretation

Katarzyna Krakowska, Paweł Marschollek, and Mariusz Chabowski: wrote the article

Maciej Malinowski and Mariusz Chabowski: critical revision of the article

Dariusz Janczak, Maciej Malinowski, and Mariusz Chabowski: final approval of article

\section{Disclosure Statement}

The authors received no financial support for this research and/or the authorship of this article. 


\section{References}

1) Olin JW, Sealove BA. Peripheral artery disease: current insight into the disease and its diagnosis and management. Mayo Clin Proc 2010; 85: 678-92.

2) Norgren L, Hiatt WR, Dormandy JA, et al. Intersociety consensus for the management of peripheral arterial disease (TASC II). Eur J Vasc Endovasc Surg 2007; 33: S1-75.

3) Owens CD, Ho KJ, Conte MS. Risk factors for failure of lower-extremity revascularization procedures: are they different for bypass and percutaneous procedures? Semin Vasc Surg 2008; 21: 143-53.

4) Urbonaviciene G, Frystyk J, Flyvbjerg A, et al. Markers of inflammation in relation to long-term cardiovascular mortality in patients with lower-extremity peripheral arterial disease. Int J Cardiol 2012; 160: 89-94.

5) Schermerhorn ML, Cronenwett JL, Baldwin JC. Open surgical repair versus endovascular therapy for chronic lower-extremity occlusive disease. Annu Rev Med 2003; 54: 269-83.

6) Endovascular treatment of peripheral artery and aortic diseases - Polish guidelines 2009. Chirurgia Polska 2009; 11: 1-12. (in Polish)

7) Adam DJ, Beard JD, Cleveland T, et al. Bypass versus angioplasty in severe ischaemia of the leg (BASIL): multicentre, randomised controlled trial. Lancet 2005; 366: 1925-34.

8) Bradbury AW, Adam DJ, Bell J, et al. Bypass versus angioplasty in severe ischaemia of the leg (BASIL) trial: An intention-to-treat analysis of amputation-free and overall survival in patients randomized to a bypass surgery-first or a balloon angioplasty-first revascularization strategy. J Vasc Surg 2010; 51: 5S-17S.

9) Dosluoglu HH, Lall P, Harris LM, et al. Long-term limb salvage and survival after endovascular and open revascularization for critical limb ischemia after adoption of endovascular-first approach by vascular surgeons. J Vasc Surg 2012; 56: 361-71.

10) Cull DL, Langan EM, Gray BH, et al. Open versus endovascular intervention for critical limb ischemia: a population-based study. J Am Coll Surg 2010; 210: 555-61, 561-3.

11) Suding PN, McMaster W, Hansen E, et al. Increased endovascular interventions decrease the rate of lower limb artery bypass operations without an increase in major amputation rate. Ann Vasc Surg 2008; 22: 195-9.

12) Brosi P, Dick F, Do DD, et al. Revascularization for chronic critical lower limb ischemia in octogenarians is worthwhile. J Vasc Surg 2007; 46: 1198-207.

13) Beard JD. Which is the best revascularization for critical limb ischemia: Endovascular or open surgery? J Vasc Surg 2008; 48: 11S-6S.

14) Bodzoń W, Krzanowski M, Maga P, et al. Endovascular treatment of severe lower leg ischemia in patients not suitable for open surgery. Chirurgia Polska 2007; 9: 18-24.

15) Morbi A, Gohel MS, Hamady M, et al. Lower-limb ischemia in the young patient: management strategies in an endovascular era. Ann Vasc Surg 2012; 26: 591-9.

16) Hynes N, Mahendran B, Manning B, et al. The influence of subintimal angioplasty on level of amputation and limb salvage rates in lower limb critical ischaemia: a 15-year experience. Eur J Vasc Endovasc Surg 2005; 30: 291-9.

17) Egorova NN, Guillerme S, Gelijns A, et al. An analysis of the outcomes of a decade of experience with lower extremity revascularization including limb salvage, lengths of stay, and safety. J Vasc Surg 2010; 51: 878-85, 885.e1.

18) Kudo T, Chandra FA, Kwun WH, et al. Changing pattern of surgical revascularization for critical limb ischemia over 12 years: endovascular vs. open bypass surgery. J Vasc Surg 2006; 44: 304-13. 